On the plastic bifurcation and post-bifurcation of axially compressed beams

Philippe Le Grognec, Anh Le van

PII:

S0020-7462(11)00004-7

DOI: doi:10.1016/j.ijnonlinmec.2011.02.001

Reference: NLM 1804

To appear in: International Journal of NonLinear Mechanics

www.elsevier.com/locate/nlm

Received date: 30 March 2010

Revised date: 10 January 2011

Accepted date: 13 February 2011

Cite this article as: Philippe Le Grognec and Anh Le van, On the plastic bifurcation and post-bifurcation of axially compressed beams, International Journal of Non-Linear Mechanics, doi:10.1016/j.ijnonlinmec.2011.02.001

This is a PDF file of an unedited manuscript that has been accepted for publication. As a service to our customers we are providing this early version of the manuscript. The manuscript will undergo copyediting, typesetting, and review of the resulting galley proof before it is published in its final citable form. Please note that during the production process errors may be discovered which could affect the content, and all legal disclaimers that apply to the journal pertain. 


\title{
On the plastic bifurcation and post-bifurcation of axially compressed beams
}

\author{
Philippe Le Grognec ${ }^{\mathrm{a}, *}$ Anh Le van ${ }^{\mathrm{b}}$ \\ ${ }^{\mathrm{a}}$ Ecole des Mines de Douai, \\ Polymers and Composites Technology 85 Mechanical Engineering Department, \\ 941 rue Charles Bourseul - BP 10838, 59508 Douai Cedex, France \\ ${ }^{\mathrm{b}} \mathrm{GeM}$ (Laboratory of Civil and Mechanical Engineering), \\ Faculty of Science - University of Nantes, \\ 2 rue de la Houssinière - BP 92208, 44322 Nantes Cedex 3, France
}

\begin{abstract}
The paper presents two new results in the domain of the elastoplastic buckling and post-buckling of beams under axial compression. (i) First, the tangent modulus critical load, the buckling mode and the initial slope of the bifurcated branch are given for a Timoshenko beam (with the transverse shear effects). The result is derived from the 3D $J_{2}$ flow plastic bifurcation theory with the von Mises yield criterion and a linear isotropic hardening. (ii) Second, use is made of a specific method in order to provide the asymptotic expansion of the post-critical branch for a Euler-Bernoulli beam, exhibiting one new non-linear fractional term. All the analytical results are validated by finite element computations.
\end{abstract}

Key words: Axially compressed beams, Bifurcation, Buckling, Elastoplasticity, Asymptotic developments

\section{Introduction}

Failure of slender or thin structures is mainly due to the buckling phenomenon and necessitates the analysis of the buckling and post-buckling behaviors for their mechanical design, namely the calculation of the critical loads, the bifurcation modes and the post-critical equilibrium branches.

* Corresponding author.

Email addresses: philippe.le.grognec@mines-douai.fr (Philippe Le Grognec), anh.le-van@univ-nantes.fr (Anh Le van). 
The buckling of a straight beam under axial compression is the most commonly studied in the literature, either in elasticity or in plasticity. The first critical load of a compressed beam in elasticity was found as the solution of an eigenvalue problem by Euler at the beginning of the eighteenth century. Much later on, in 1945, Koiter succeeded in describing the elastic post-bifurcation behavior using an asymptotic power expansion. At the same time, he also achieved the imperfection sensitivity analysis for an elastic buckled beam. On the other hand, the plastic bifurcation analysis, even on a simple model such as a beam, makes much less progress than in elasticity. It still is the subject of a lot of recent theoretical as well as experimental investigations.

In the sequel, we will briefly recall the main results available in the literature on the plastic bifurcation, with no attempt to make an exhaustive review (see, for instance, the survey paper of Sewell (1972) for a general review on plastic buckling). The interested reader can find more details on the theoretical developments and more complete states-of-art in the quoted references below.

The pioneering works on the critical load of an elastoplastic beam under axial compression dated back to the end of the nineteenth century and were conducted by Engesser, Considère, and later by von Karman. Yet, the early results before the 1940's were not quite correct or properly justified. Some authors derived the tangent modulus critical load by discarding the unloading possibility in the structure; others obtained the reduced modulus critical load by assuming that the bifurcation takes place at constant load as in elasticity. The first significant result is due to Shanley (1947), who provided a rational explanation for the plastic buckling of the so-called Shanley's column, which had been introduced by von Karman in elasticity. The considered model is a rigid rod with two degrees of freedom; it is supported by two elastoplastic springs at the bottom and subjected to an axial compressive force at the tip. This discrete model supposedly able to reproduce the behavior of a beam cross-section did lead to results which are qualitatively similar to those of a continuum structure under plastic buckling. Shanley thus provided a satisfactory answer to the value and the nature of the first critical load. In discrete or continuous structures, the first bifurcation occurs at the tangent modulus critical load, giving rise to an incipient unloaded zone and an increasing load during the initial post-bifurcation. Hill (1958) extended these results to a 3D continuum by using the concept of "comparison elastic solid". He examined the uniqueness and stability criteria, and pointed out the difference between bifurcation and stability.

As regards the plasticity theory to be used for solving the plastic buckling problem, one can choose between the $J_{2}$ deformation and flow theories. These two theories may yield different critical values and they coexist as they have each their own advantages and drawbacks. The deformation theory, although it does not take into account the elastic unloading possibility, yields critical 
loads that compare best with the experiments, whereas the flow theory generally overpredicts the critical values. The discrepancy - known as the plastic buckling paradox - can be accounted for and quantified through an analysis of imperfection sensitivity, as can be found in Durban (1998).

In order to study the plastic post-buckling, Hutchinson (1974) extended Koiter's results in elasticity by using a series expansion with fractional exponents. He improved the Shanley model by replacing the above-mentioned two springs system with a uniform distribution of springs. He also studied compressed beams and some more general cases by assuming a homogeneous prebifurcation state. The series expansion with fractional exponents was derived laboriously and is only valid in initial post-bifurcation. Much later, Cimetière, El Koulani and Léger (Cimetière, El Koulani and Léger, 1994; El Koulani, 1996; El Koulani and Léger, 1996, 1997) thoroughly solved the bifurcation problem for the Shanley column and the compressed beam, and first provided the necessary theoretical ingredients such as the validity, the convergence of the previous expansion and the existence of the post-critical branches. In the beam case, the Hutchinson asymptotic expansion giving the load parameter versus the perturbation parameter (i.e. the bifurcation mode amplitude) was limited to the first non-linear term, because of the complexity of the coordinate system used to describe the evolution of the unloaded zone. In the 1980's, Nguyen and Stolz (1985) and Akel (1987) reformulated the problem within the framework of the generalized standard materials and expanded both the load and the perturbation parameter in power series of a "time" parameter. They showed that these simple power series yield the same series with fractional exponents as obtained by Hutchinson.

Another significant result in plastic buckling problems is the existence of continua of bifurcation points, which was discovered by Cimetière (1987) when dealing with the case of compressed rectangular plates. These continua enable the structure to bifurcate within intervals of critical loads, by continuously modifying the unloaded zone and the structural stiffness. Each continuous range of bifurcation points (one per mode) observed in plasticity spreads from the tangent modulus critical value $\lambda_{T}$ to the elastic one $\lambda_{E}$. It contains a particular intermediate value which is the reduced modulus critical load $\lambda_{R}$ corresponding to a constant loading bifurcation. In general, the plastic buckling may appear in three different ways, depending on the yield strength value $\lambda_{y}$. If the yield force precedes the first continuous range $\left(\lambda_{y} \leqslant \lambda_{T}\right)$, the plastic buckling occurs at the tangent modulus critical load. If $\lambda_{y} \geqslant \lambda_{E}$, the buckling is purely elastic. Eventually, in the intermediate case when $\lambda_{T} \leqslant \lambda_{y} \leqslant \lambda_{E}$, referred to as the plastic breakdown (Akel, 1987), the plastic buckling occurs at the plastification load $\lambda_{y}$ precisely.

Most results were obtained for the plastic bifurcation at the tangent modulus critical value. The load $\lambda$ acts as a bifurcation parameter; the perturbation 
parameter denoted by $\xi(\xi \geqslant 0)$ can be viewed as a measure of the difference between the fundamental and post-buckling solutions. The asymptotic expansion proposed by Hutchinson (1974) for a general structure reads:

$$
\lambda=\lambda_{T}+\lambda_{1} \xi+\lambda_{2} \xi^{1+\beta}+\ldots
$$

where $\lambda_{1}$ is generally positive, thus verifying the hypothesis of an increasing bifurcation load, $\lambda_{2}$ is negative, and the exponent $\beta$ is a rational number such that $0<\beta<1$. The $\lambda_{2}<0$ condition leads one to predict a load limit point on the bifurcated branch, i.e. an upper bound for the strength of the elastoplastic structure in the post-critical behavior. In the case of a beam with a circular cross-section, the unloading initiates at a single point and $\beta$ is $1 / 3$. For a rectangular cross-section, a whole line is simultaneously unloaded and $\beta$ is $2 / 5$. It is noteworthy that in the case of a symmetric section, the previous expansion can be used with $\xi<0$ by replacing $\xi$ with $-\xi$ in relation (1), thus making $\lambda(\xi)$ an even function; conversely, this procedure does not work for a non-symmetric section, as mentionned by Tvergaard and Needleman (1975).

More recently, Cheng, Lu and Fang (1995) proposed a simpler approach in order to obtain the second fractional term in the beam case with a rectangular cross-section (the method can be applied to plates and shells as well). By means of a new coordinate system which is not directly related to the geometry of the unloaded zone, they found the known first three terms in relation (1) again, determined the fourth term, and showed that one cannot go further without breaking the continuity of the solution. Writing the virtual work principle at different "time" orders enabled them to obtain a new equilibrium branch, bifurcating at the tangent modulus critical value, with a new maximum load, much greater than that found in earlier works. Later on, the same authors extended their analysis by adding a geometric imperfection (Cheng, Fang and Lu, 1998). They emphasized that the elastic unloading prevails over geometric non-linearities, at least in a first deformation stage. Moreover, they showed that a growing imperfection modifies the previous maximum load value and finally makes it completely disappear.

Whilst the bifurcation occurs at the tangent modulus critical load in practice, it can theoretically take place at any other point of the continuous range. Yet, only a few qualitative results are available for the bifurcated branches arising from the critical loads other than the tangent modulus one. During initial post-bifurcation, the load increases when bifurcation occurs between $\lambda_{T}$ and $\lambda_{R}$ and decreases between $\lambda_{R}$ and $\lambda_{E}$. By using a stability criterion based on a plastic potential, Nguyen (1984) showed that the bifurcated branches with an increasing load $\left(\lambda_{c} \in\left[\lambda_{T}, \lambda_{R}\right]\right)$ have a stable behavior and the postbuckling behavior far from the fundamental solution depends on the geometric non-linearities taken into account. 


\section{Scope and outline of the paper}

This paper is devoted to two new theoretical issues of the plastic bifurcation and post-bifurcation of axially compressed beams. The beams have no geometric imperfection and the bifurcation is assumed to occur at the tangent modulus value.

(i) Section 3 deals with the plastic bifurcation of a Timoshenko beam, where the transverse shear effects are taken into account. The analysis is based on the $3 \mathrm{D}$ plastic bifurcation theory, using the $J_{2}$ flow theory assuming the von Mises yield criterion and a linear isotropic hardening. This $3 \mathrm{D}$ approach is a general and efficient way to cope with structures subjected to a uniform uniaxial precritical stress state. It was successfully applied to the case of compressed plates and cylinders in an earlier study (Le Grognec and Le van, 2009). Here, the same approach will provide the tangent modulus critical load, the buckling mode and the initial slope of the bifurcated branch for a Timoshenko beam. The analytical values will be displayed together with finite element results for a beam with a hollow rectangular section, where the transverse shear effects are significant.

(ii) In Section 4, we will limit ourselves to Euler-Bernoulli beams and aim to go further in the post-bifurcation analysis stemming from the tangent modulus bifurcation. The goal will be achieved by using a specific method which provides both the fractional expansion of the post-critical branch containing one new non-linear term and the shape of the elastic unloading region in the beam during the bifurcation process. All the analytical solutions presented will be compared to finite element results.

\section{Plastic bifurcation analysis of a Timoshenko beam}

\subsection{Critical load and bifurcation mode}

Let us consider a straight Timoshenko beam subjected to an axial compressive force which leads to the buckling in the plastic regime. The critical load and the bifurcation mode of the beam will be derived from a 3D approach which is in essence similar to that presented in Le Grognec and Le van (2009) for the plastic buckling of plates and cylinders. The ingredients in the quoted reference will be summarized below and it will be shown how the 3D approach can also be applied to the beam case.

The theory is developed using a total Lagrangian formulation where the beam 
is seen as a 3D body. In the reference configuration, the beam occupies a cylindrical volume $\Omega_{o}$ of constant cross-section area $S$ and length $L$. In the elastic regime, the material is assumed to be isotropic, defined by the fourth-order elasticity tensor $\mathbf{D}$ whose components in an orthonormal basis are $D_{i j k l}=$ $\bar{\lambda} \delta_{i j} \delta_{k l}+\mu\left(\delta_{i k} \delta_{j l}+\delta_{i l} \delta_{k j}\right)$, where $\delta_{i j}$ is the Kronecker symbol, $\bar{\lambda}$ and $\mu$ are the Lamé constants. Use is also made of the Young's modulus $E$ and the Poisson's ratio $\nu$ related to $\bar{\lambda}$ and $\mu$ by standard relations $\bar{\lambda}=\frac{E \nu}{(1+\nu)(1-2 \nu)}$ and $\mu=\frac{E}{2(1+\nu)}$. We assume that the plastic threshold is defined by the von Mises yield function with a linear isotropic hardening:

$$
f(\Sigma, A)=\sqrt{\frac{3}{2} \Sigma^{d}: \Sigma^{d}}-\sigma_{0}-A \quad A=H p
$$

where $\boldsymbol{\Sigma}$ denotes the second Kirchhoff stress tensor (symmetric), $\boldsymbol{\Sigma}^{d}$ its deviatoric part, $\sigma_{0}$ the yield stress, $H$ the isotropic hardening modulus (constant) and $p$ the equivalent plastic strain.

The material tangent elastoplastic tensor is:

$$
\mathbf{D}^{p}=\frac{\partial \boldsymbol{\Sigma}}{\partial \mathbf{E}}=\mathbf{D}-\frac{\mathbf{D}: \frac{\partial f}{\partial \boldsymbol{\Sigma}} \otimes \frac{\partial f}{\partial \boldsymbol{\Sigma}}: \mathbf{D}}{H+\frac{\partial f}{\partial \boldsymbol{\Sigma}}: \mathbf{D}: \frac{\partial f}{\partial \boldsymbol{\Sigma}}}
$$

where $\mathbf{E}$ denotes the Green strain tensor and the tensor product $\otimes$ of two second-order tensors $\mathbf{S}$ and $\mathbf{T}$ is defined by $(\mathbf{S} \otimes \mathbf{T})_{i j k l}=S_{i j} T_{k l}$. Relation (3) can be recast as:

$$
\mathbf{D}^{p}=\mathbf{D}-\mathbf{N} \otimes \mathbf{N}
$$

where the symmetric tensor $\mathbf{N}$ is:

$$
\mathbf{N}=\frac{\mathbf{D}: \frac{\partial f}{\partial \boldsymbol{\Sigma}}}{\sqrt{H+\frac{\partial f}{\partial \boldsymbol{\Sigma}}: \mathbf{D}: \frac{\partial f}{\partial \boldsymbol{\Sigma}}}}
$$

One then derives the nominal tangent elastoplastic tensor by:

$$
\mathbf{K}^{p}=\frac{\partial \boldsymbol{\Pi}}{\partial \mathbf{F}}=\mathbf{F} \cdot \frac{\partial \boldsymbol{\Sigma}}{\partial \mathbf{E}} \cdot \mathbf{F}^{T}+(\mathbb{I} \cdot \boldsymbol{\Sigma})^{T}
$$

In the previous equation, $\mathbf{F}$ is the deformation gradient, $\Pi=\mathbf{F} . \boldsymbol{\Sigma}$ the first Kirchhoff stress tensor (non-symmetric), II represents the fourth-order unit tensor $\left(\mathbb{I}_{i j k l}=\delta_{i l} \delta_{k j}\right)$ and the superscript ${ }^{T}$ the major transposition of a fourthorder tensor $\left(\left(\mathbf{D}^{T}\right)_{i j k l}=D_{k l i j}\right)$. Like (4), Equation (6) can be rewritten as:

$$
\mathbf{K}^{p}=\mathbf{K}^{e}-\mathbf{M}^{T} \otimes \mathbf{M}
$$

with:

$$
\mathbf{K}^{e}=\mathbf{F} \cdot \mathbf{D} \cdot \mathbf{F}^{T}+(\mathbb{I} \cdot \boldsymbol{\Sigma})^{T} \quad \mathbf{M}=\mathbf{N} \cdot \mathbf{F}^{T}
$$


Now, let us assume that there exists a fundamental equilibrium path $\lambda \mapsto$ $\mathbf{U}_{f}(\lambda)$, which is the displacement solution of the elastoplastic problem under an increasing load factor $\lambda(\dot{\lambda}>0)$, and that there exists a bifurcated solution $\mathrm{U}$ at a critical time $t_{c}$ corresponding to $\lambda_{c}=\lambda\left(t_{c}\right)$. Furthermore, the yield stress $\sigma_{0}$ is assumed to be small enough for the plastic strains to appear before the tangent modulus critical load is reached.

The bifurcated branch is described by an asymptotic expansion with respect to the perturbation parameter $\xi(\xi \geqslant 0)$ :

$$
\begin{aligned}
& \lambda=\lambda_{c}+\lambda_{1} \xi+o(\xi) \\
& \mathbf{U}=\mathbf{U}_{f}(\lambda)+\xi \mathbf{X}+o(\xi)
\end{aligned}
$$

Let us make the following assumption:

\section{Assumption 1}

- At critical time $t_{c}$, the plastic zone $\Omega^{p}\left(t_{c}\right)$ corresponding to the bifurcated solution is equal to that of the fundamental solution $\Omega_{f}^{p}\left(t_{c}\right)$, i.e. the bifurcation takes place at the tangent modulus critical load with incipient unloading.

- The whole solid is plastified on the fundamental branch:

$$
\Omega_{f}^{p}\left(t_{c}\right)=\Omega_{o}
$$

which implies that the nominal tangent elastoplastic tensor is equal to $\mathbf{K}^{p}$ throughout the solid

With the above assumption, the critical load $\lambda_{c}=\lambda_{T}$ and the bifurcation mode $\mathbf{X}$ are obtained by solving the following bifurcation equation (Hutchinson, 1972; Nguyen, 2000):

$$
\forall \delta \mathbf{U}, \quad \int_{\Omega_{o}} \nabla^{T} \delta \mathbf{U}: \mathbf{K}^{p}\left(\mathbf{U}_{f}\left(\lambda_{c}\right)\right): \nabla \mathbf{X} d \Omega_{o}=0
$$

We shall now derive more explicit expressions of the above results by exploiting the uniaxial stress state in the compressed beam at hand. Let the beam be subjected in the pre-critical state to a nominal axial compressive stress $\Pi_{X X}=-\lambda<0$, so that the first Kirchhoff stress tensor $\Pi$ is expressed in an orthonormal basis $\left(\mathbf{e}_{1}, \mathbf{e}_{2}, \mathbf{e}_{3}\right)$ as (we indifferently denote $(X, Y, Z)$ and 
$(1,2,3))$ :

$$
\boldsymbol{\Pi}=-\lambda \mathbf{e}_{1} \otimes \mathbf{e}_{1}=\left[\begin{array}{ccc}
-\lambda & 0 & 0 \\
0 & 0 & 0 \\
0 & 0 & 0
\end{array}\right] \quad(\lambda>0)
$$

Let us make the following assumption, which is usually satisfied in practice:

Assumption 2. The pre-critical deformations are small:

$$
\|\nabla \mathbf{U}\| \ll 1
$$

Thus, the stress tensor $\boldsymbol{\Sigma}$ writes:

$$
\Sigma=\mathbf{F}^{-1} \cdot \Pi \approx \Pi
$$

The tensor $\mathbf{N}$ in Equation (5) then simplifies as follows:

$$
\mathbf{N}=\frac{\mu}{\sqrt{H+3 \mu}}\left(\mathbf{I}-3 \mathbf{e}_{1} \otimes \mathbf{e}_{1}\right)=\frac{\mu}{\sqrt{H+3 \mu}}\left[\begin{array}{rrr}
-2 & 0 & 0 \\
0 & 1 & 0 \\
0 & 0 & 1
\end{array}\right]
$$

Hence, the material tangent elastoplastic tensor in Equation (4) reads:

$$
\mathbf{D}^{p}=\mathbf{D}-\frac{\mu^{2}}{H+3 \mu}\left(\mathbf{I}-3 \mathbf{e}_{1} \otimes \mathbf{e}_{1}\right) \otimes\left(\mathbf{I}-3 \mathbf{e}_{1} \otimes \mathbf{e}_{1}\right)
$$

The components of $\mathbf{D}^{p}$ in the orthonormal basis $\left(\mathbf{e}_{1}, \mathbf{e}_{2}, \mathbf{e}_{3}\right)$ are:

$$
\begin{aligned}
D_{1111}^{p} & =\bar{\lambda}+2 \mu-\frac{4 \mu^{2}}{H+3 \mu} & & D_{2233}^{p}=\bar{\lambda}-\frac{\mu^{2}}{H+3 \mu} \\
D_{2222}^{p} & =\bar{\lambda}+2 \mu-\frac{\mu^{2}}{H+3 \mu} & & D_{1133}^{p}=\bar{\lambda}+\frac{2 \mu^{2}}{H+3 \mu} \\
D_{3333}^{p} & =\bar{\lambda}+2 \mu-\frac{\mu^{2}}{H+3 \mu} & & D_{1122}^{p}=\bar{\lambda}+\frac{2 \mu^{2}}{H+3 \mu} \\
D_{1212}^{p} & =D_{1313}^{p}=D_{2323}^{p}=\mu & &
\end{aligned}
$$

The other components are either zero or derived from Equation (17) using both major and minor symmetries of tensor $\mathbf{D}^{p}\left(D_{i j k l}^{p}=D_{k l i j}^{p}=D_{j i k l}^{p}=D_{i j l k}^{p}\right)$.

The nominal tangent elastoplastic tensor in Equation (7) becomes:

$$
\mathbf{K}^{p} \approx \frac{\partial \boldsymbol{\Sigma}}{\partial \mathbf{E}}+(\mathbb{I} . \boldsymbol{\Sigma})^{T}=\mathbf{D}^{p}-\lambda \mathbf{e}_{i} \otimes \mathbf{e}_{1} \otimes \mathbf{e}_{1} \otimes \mathbf{e}_{i}
$$


which is independent of the spatial coordinates.

Furthermore, when dealing with 1D models like beams, ad hoc assumptions are usually added in order to enforce some specific stress state in the body. Namely, the transverse normal material stresses are assumed to be zero: $\Sigma_{22}=\Sigma_{33}=0$. Taking into account these assumptions leads one to replace tensor $\mathbf{D}^{p}$ in (16) with the reduced tensor $\mathbf{C}^{p}$ defined as:

$$
\begin{array}{r}
C_{i j k l}^{p}=D_{i j k l}^{p}+\frac{D_{i j 22}^{p}\left(D_{2233}^{p} D_{33 k l}^{p}-D_{333}^{p} D_{22 k l}^{p}\right)+D_{i j 33}^{p}\left(D_{3322}^{p} D_{22 k l}^{p}-D_{2222}^{p} D_{33 k l}^{p}\right)}{D_{2222}^{p} D_{3333}^{p}-D_{2233}^{p} D_{3322}^{p}} \\
(i, j) \neq(2,2),(3,3) \quad(k, l) \neq(2,2),(3,3)
\end{array}
$$

It can be readily checked that tensor $\mathbf{C}^{p}$ has the major and both minor symmetries. In the sequel, we only need the following reduced moduli (and their equivalents obtained by major or minor symmetries):

$$
C_{1111}^{p}=E_{T} \quad C_{1212}^{p}=C_{1313}^{p}=C_{2323}^{p}=\mu
$$

where $E_{T}$ is the tangent elastoplastic modulus related to Young's modulus $E$ and the isotropic hardening modulus $H$ by $\frac{1}{E_{T}}=\frac{1}{E}+\frac{1}{H}$.

Eventually, the bifurcation equation (11) writes in the uniaxial stress case:

$$
\forall \delta \mathbf{U}, \quad \int_{\Omega_{o}} \nabla^{T} \delta \mathbf{U}:\left(\mathbf{C}^{p}-\lambda_{c} \mathbf{e}_{i} \otimes \mathbf{e}_{1} \otimes \mathbf{e}_{1} \otimes \mathbf{e}_{i}\right): \nabla \mathbf{X} d \Omega_{o}=0
$$

Let us consider the bending problem in the xy-plane of a beam built-in at end $X=0$ and compressed by load $\lambda$ at end $X=L$. The Timoshenko kinematics is defined by two scalar displacement fields $U(X, t)$ and $V(X, t)$, respectively the axial and transverse displacements of the centroid axis of the beam, and the cross-section rotation $\theta(X, t)$, independent of deflection $V$. When the beam buckles from the straight position (the fundamental solution) to a bent shape, the expressions for the bifurcation mode $\mathbf{X}$ and the displacement variation $\delta \mathbf{U}$ are chosen according to the Timoshenko kinematics:

$$
\mathbf{X}=\left|\begin{array}{l}
\mathcal{U}-Y \Theta \\
\mathcal{V} \\
0
\end{array} \quad \delta \mathbf{U}=\right| \begin{aligned}
& \delta U-Y \delta \theta \\
& \delta V \\
& 0
\end{aligned}
$$


The bifurcation equation (21) becomes:

$$
\begin{aligned}
& \forall \delta U, \delta V, \delta \theta \text { satisfying } \delta U(0)=\delta V(0)=\delta \theta(0)=0, \\
& \int_{\Omega_{o}}\left[E_{T}\left(\mathcal{U},{ }_{X}-Y \Theta,_{X}\right)\left(\delta U,{ }_{X}-Y \delta \theta,_{X}\right)\right. \\
& -\mu \mathcal{V},_{X} \delta \theta-\mu \Theta \delta V,_{X}+\mu \mathcal{V},_{X} \delta V,_{X}+\mu \Theta \delta \theta \\
& \left.-\lambda_{c}\left(\mathcal{U},,_{X}-Y \Theta,_{X}\right)\left(\delta U,_{X}-Y \delta \theta,_{X}\right)-\lambda_{c} \mathcal{V},,_{X} \delta V,_{X}\right] d \Omega_{o}=0
\end{aligned}
$$

First, integrating over the cross-section, then integrating by parts with respect to $X$ and eliminating negligible higher-order terms yields three local equations for the components $\mathcal{U}, \mathcal{V}$ and $\Theta$ of the eigenmode:

$$
\begin{aligned}
& E_{T} S \mathcal{U},_{X X}=0 \\
& k \mu S\left(\Theta,_{X}-\mathcal{V},{ }_{X X}\right)+\lambda_{c} S \mathcal{V},{ }_{X X}=0 \\
& E_{T} I \Theta,_{X X}+k \mu S\left(\mathcal{V},{ }_{X}-\Theta\right)=0
\end{aligned}
$$

together with the stress boundary conditions at the end $X=L: \mathcal{U},{ }_{X}(L)=0$, $k \mu S\left(\Theta(L)-\mathcal{V},_{X}(L)\right)+\lambda_{c} S \mathcal{V},_{X}(L)=0$ and $\Theta,_{X}(L)=0$, where $S$ denotes the cross-section area, $I$ the second moment of area and $k$ is introduced as the transverse shear correction factor depending on the cross-section of the beam.

Taking into account $\mathcal{U}(0)=\mathcal{V}(0)=\Theta(0)=0$, one obtains the first critical load and the corresponding bifurcation mode (Le Grognec and Le van, 2004):

$$
\lambda_{C} S=\lambda_{T} S=\frac{\frac{\pi^{2} E_{T} I}{4 L^{2}}}{1+\frac{\pi^{2} E_{T} I}{4 k \mu S L^{2}}}
$$

$$
\left\{\begin{array}{l}
\mathcal{U}=0 \\
\mathcal{V}=C\left(1+\frac{\pi^{2} E_{T} I}{4 k \mu S L^{2}}\right)\left(1-\cos \frac{\pi X}{2 L}\right) \quad(C \text { is an arbitrary constant }) \\
\Theta=\frac{\pi C}{2 L} \sin \frac{\pi X}{2 L}
\end{array}\right.
$$

Similar differential equations and solutions for the $J_{2}$ deformation theory can be obtained from the above results, provided the tangent moduli are replaced by the equivalent secant moduli. 


\subsection{Initial slope of the bifurcated branch}

The initial slope of the bifurcated branch at the critical point, equal to coefficient $\lambda_{1}$ in the expansion (9), can be expressed under the following assumption:

\section{Assumption 3}

- $\lambda_{c}$ is a simple eigenvalue.

- The so-called transversality coefficient $\operatorname{Tr}$ is positive:

$$
T r \doteq-\int_{\Omega_{o}} \nabla^{T} \mathbf{X}:{\frac{d \mathbf{K}^{p}\left(\mathbf{U}_{f}(\lambda)\right)}{d \lambda}}_{\mid \lambda=\lambda_{c}}: \nabla \mathbf{X} d \Omega_{o}>0
$$

- The plastic zone corresponding to the fundamental solution at the critical time $\Omega_{f}^{p}\left(t_{c}\right)\left(=\Omega_{o}\right)$ is active (i.e. there is loading and no neutral loading).

- $\exists \Delta>0, \forall X \in \Omega_{f}^{p}\left(t_{c}\right), \mathbf{M}\left(X, \lambda_{c}\right): \nabla \mathbf{U}_{f, \lambda}\left(X, \lambda_{c}\right) \geqslant \Delta>0$, where $X \in \Omega_{o}$ denotes the position of the current particle in the reference configuration

Under this assumption, one has an angular bifurcation and the initial slope $\lambda_{1}$ is given by:

$$
\lambda_{1}=\max _{X \in \Omega_{f}^{p}\left(t_{c}\right)}\left(-\frac{\mathbf{M}\left(X, \lambda_{c}\right): \nabla \mathbf{X}}{\mathbf{M}\left(X, \lambda_{c}\right): \nabla \mathbf{U}_{f, \lambda}\left(X, \lambda_{c}\right)}\right)
$$

In fact, it can be readily checked that the transversality condition (27) is always satisfied in the uniaxial stress state. From expression (18) for the nominal tangent elastoplastic tensor, the transversality coefficient $\operatorname{Tr}$ in (27) is reduced to:

$$
\operatorname{Tr}=\int_{\Omega_{0}}\left|\mathbf{X}_{, 1}\right|^{2} d \Omega_{o}
$$

whence, by (26), $\operatorname{Tr}$ is strictly positive.

Furthermore, from Equation (8) and assumption (13), one has $\mathbf{M}=\mathbf{N} \cdot \mathbf{F}^{T} \approx$ N. Hence:

$$
\begin{aligned}
\mathbf{M}: \nabla \mathbf{X} & =\frac{\mu}{\sqrt{H+3 \mu}}\left(\mathbf{I}-3 \mathbf{e}_{1} \otimes \mathbf{e}_{1}\right): \nabla \mathbf{X} \\
& =\frac{\mu}{\sqrt{H+3 \mu}}\left(\operatorname{div} \mathbf{X}-3(\nabla \mathbf{X})_{11}\right)
\end{aligned}
$$

Accordingly, the initial slope of the bifurcated branch (28) becomes:

$$
\lambda_{1}=\max _{X \in \Omega_{o}}\left(-\frac{3(\nabla \mathbf{X})_{11}-\operatorname{div} \mathbf{X}}{3\left(\nabla \mathbf{U}_{f, \lambda}^{c}\right)_{11}-\operatorname{div}\left(\mathbf{U}_{f, \lambda}^{c}\right)}\right)
$$

where $\mathbf{U}_{f, \lambda}^{c}$ denotes the differentiation $\mathbf{U}_{f, \lambda}(X, \lambda)$ evaluated at $\lambda=\lambda_{c}$. 
The fundamental solution for the entirely plastified beam subjected to load $\lambda \geqslant \sigma_{0}$ has the same form as in small deformations:

$$
\mathbf{U}_{f}=-\left(\frac{\lambda-\sigma_{0}}{E_{T}}+\frac{\sigma_{0}}{E}\right) X \mathbf{x}
$$

From Equation (31), one then can derive the initial slope for a beam with a rectangular cross-section of height $e$ in the $\mathbf{y}$-direction, choosing the value of $C$ equal to $e / 2$ in expression $(26)$ for $\mathcal{V}$ :

$$
\lambda_{1} S=\frac{3 \pi^{2} E_{T} I}{4 L^{2}}=3\left(1+\frac{\pi^{2} E_{T} I}{4 k \mu S L^{2}}\right) \lambda_{T} S
$$

REMARK. Making $k$ tend to infinity in relations (25), (26) and (33) yields the well-known expressions for the first critical load, the corresponding bifurcation mode and the initial slope in the case of a Euler-Bernoulli beam:

$$
\begin{gathered}
\lambda_{c} S=\lambda_{T} S=\frac{\pi^{2} E_{T} I}{4 L^{2}} \\
\left\{\begin{array}{l}
\mathcal{U}=0 \\
\mathcal{V}=C\left(1-\cos \frac{\pi X}{2 L}\right) \quad(C \text { is an arbitrary constant }) \\
\lambda_{1} S=\frac{3 \pi^{2} E_{T} I}{4 L^{2}}=3 \lambda_{T} S
\end{array}\right.
\end{gathered}
$$

The Timoshenko beam model provides a slightly smaller critical load than the Euler-Bernoulli model, whereas the initial slope is the same

\subsection{Finite element validation}

In order to check the plastic buckling load expression (25) for a Timoshenko beam, a numerical computation is performed by means of a home-made finite element program. Developed within a total Lagrangian framework, the program encompasses finite plasticity and geometric non-linearities (finite displacements and rotations) in order to deal with the plastic buckling and advanced post-critical behavior of general structures. For the computation of interest, the beam is discretized using a finite rotation shell finite element which is described in full details in Le Grognec and Le van (2008) in the context of cylindrical shells under axial compression.

Consider a beam of length $L=1 \mathrm{~m}$ in the $\mathbf{x}$-direction, with a rectangular hollow cross-section of height $e=250 \mathrm{~mm}$ in the $\mathbf{y}$-direction and width $b=$ 
$200 \mathrm{~mm}$ in the z-direction. The wall thickness is 2, 3 or $5 \mathrm{~mm}$. The Young's modulus is $E=2.110^{11} \mathrm{~Pa}$, the Poisson's ratio $\nu=0.3$ and the yield stress $\sigma_{0}=5.10^{7} \mathrm{~Pa}$. The isotropic hardening modulus is $H=1.510^{11} \mathrm{~Pa}$, hence the tangent elastoplastic modulus $E_{T}=\frac{E H}{E+H}=8.7510^{10} \mathrm{~Pa}$ (the hardening modulus $H$ has been given a high value in order to separate the different continua of bifurcation points and thus help us to analyze the numerical results more easily). The shear correction factor introduced in Equation (24) takes the value $k=5 / 12 \approx 0.417$ usually chosen for square hollow sections. Some rotational degrees of freedom of the shell elements are prescribed to zero in order to prevent a local buckling mode and enforce the global mode to take place in the $\mathbf{x y}$-plane. Figure 1 shows the buckling mode obtained by the finite element computation.

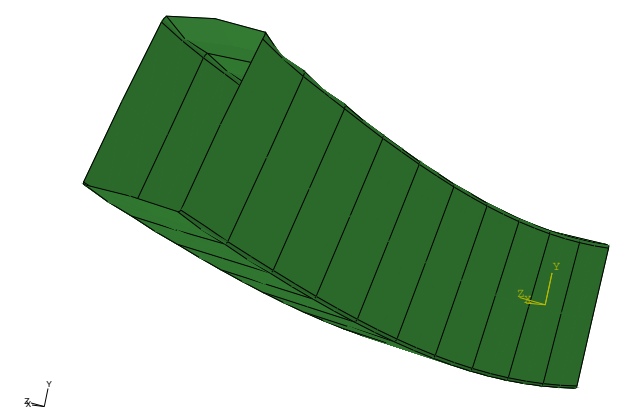

Fig. 1. Plastic buckling mode of a compressed Timoshenko beam with a rectangular hollow cross-section, obtained by the finite element method

The analytical critical loads obtained from the Timoshenko model (25) and the Euler-Bernoulli model (34) are compared with the finite element values in Table 1 and Figure 2 for various thicknesses. The term $\frac{\pi^{2} E_{T} I}{4 k \mu S L^{2}}$ in $(25)$ responsible for the difference between the two kinematical models does not depend on the thickness of the thin-walled beam and is about $6.3 \%$. It is found that for cross-section geometries such as the one considered here - for which the transverse effects are significant - the Timoshenko model gives better results and is more relevant than the Euler-Bernoulli one.

\begin{tabular}{|c|c|c|c|c|}
\hline $\begin{array}{c}\text { Thickness } \\
(m m)\end{array}$ & $\begin{array}{c}\lambda_{c} S(N) \\
(\text { Euler-Bernoulli) }\end{array}$ & $\begin{array}{c}\lambda_{c} S(N) \\
(\text { Timoshenko) }\end{array}$ & $\begin{array}{c}\lambda_{c} S(N) \\
(\mathbf{F E})\end{array}$ & $\begin{array}{c}\text { Error (\%) } \\
\text { (Timoshenko vs. FE) }\end{array}$ \\
\hline 2 & $3.82310^{6}$ & $\mathbf{3 . 5 9 6} \mathbf{1 0}^{\mathbf{6}}$ & $\mathbf{3 . 5 9 6} \mathbf{1 0}^{\mathbf{6}}$ & 0.0005 \\
\hline 3 & $5.73510^{6}$ & $\mathbf{5 . 3 9 5} \mathbf{1 0}^{\mathbf{6}}$ & $\mathbf{5 . 4 4 4} \mathbf{1 0}^{\mathbf{6}}$ & 0.9 \\
\hline 5 & $9.55810^{6}$ & $\mathbf{8 . 9 9 1} \mathbf{1 0}^{\mathbf{6}}$ & $\mathbf{9 . 1 1} \mathbf{1 0}^{\mathbf{6}}$ & 1.3 \\
\hline
\end{tabular}

Table 1

Critical loads of a rectangular hollow beam under axial compression with various thicknesses 


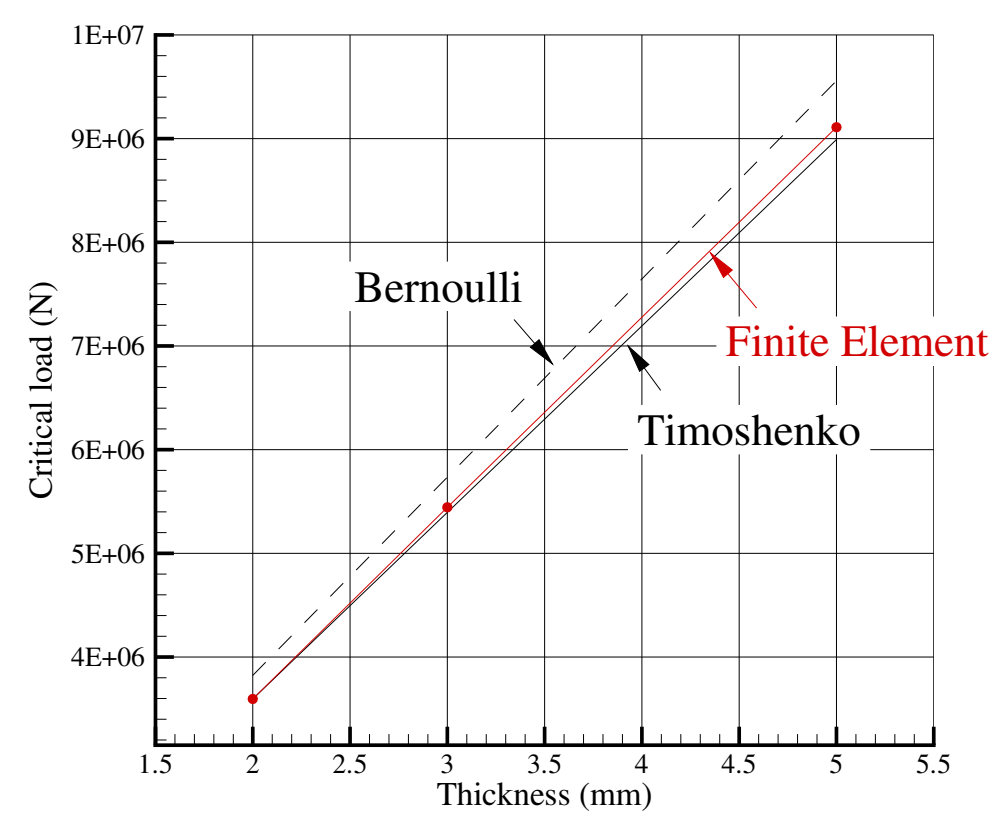

Fig. 2. Comparison between numerical and analytical critical values with or without transverse shear effect

\section{Plastic post-bifurcation analysis of a Euler-Bernoulli beam}

The aim of this section is to go further in the expansion (9) and investigate the post-bifurcation behavior of the beam.

The post-bifurcation analysis will be carried out for the Euler-Bernoulli beam model only; although the Timoshenko model could be treated using the same line of argument, the corresponding equations would be much more intricate. Also, for the sake of definiteness, we will focus on the first bifurcation mode arising from the tangent modulus critical load of a beam, built-in at end $X=0$, with a rectangular cross-section of height $e$ in the $\mathbf{y}$-direction and width $b$ in the $\mathbf{z}$-direction, see Figure 3.

A generic approach will be presented, which enables one to obtain one more term in comparison with Hutchinson's asymptotic expansion of the bifurcated branch.

Let the compressed beam buckle into a bent position with negative $Y$-values. The elastic unloaded zone, reduced to a single point in the $\mathbf{x y}$-plane at the critical time, is assumed to spread out into a connected region separated from the plastic zone by a smooth boundary defined by $Y=d(X, t), X \in[0, a]$, as shown in Figure 3. 

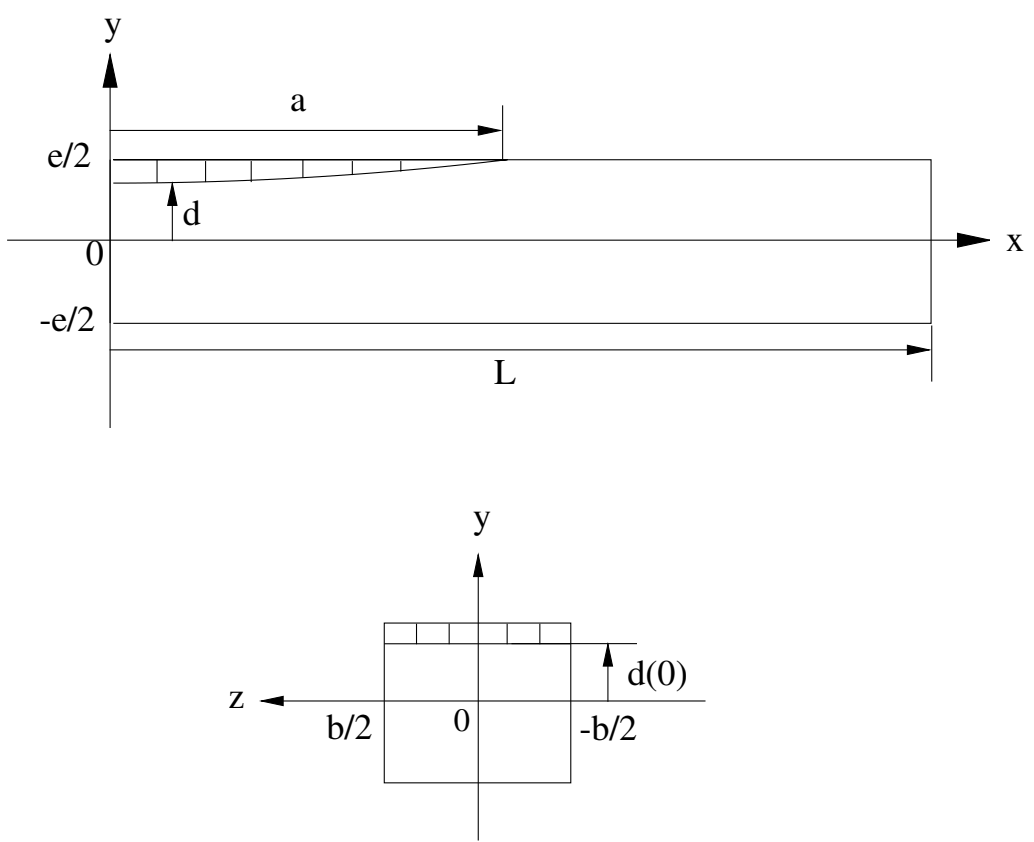

Cross-section at $\mathrm{x}=0$

Fig. 3. Unloaded zone (shaded) during the plastic buckling of a cantilever beam 4.1 Power series expansions for the load and the deflection

According to the procedure described in Nguyen (2000), the four following operations are performed:

(a) To define $v(X, t)=V(X, t)-V(L, t)$.

(b) To assume a non-constant load factor: $\dot{\lambda} \neq 0$ (in this section, $\lambda$ denotes the axial force instead of the axial stress) and introduce the function $r(X, t)$ defined by:

$$
\dot{v}(X, t)=\dot{\lambda}(t) r(X, t)
$$

(c) To use the following change of variables, which enables one to eliminate parameter $a$ and write the equations on fixed space intervals:

- for $X \in] 0, a\left[: \quad X=a s \quad \leftrightarrow \quad s=\frac{X}{a}\right.$ with $\left.s \in\right] 0,1[$

- for $X \in] a, L\left[: X=a+(L-a)(s-1) \quad \leftrightarrow \quad s=\frac{X-a}{L-a}+1\right.$ with $\left.s \in\right] 1,2[$

(d) To choose as the kinematic "time" $t$ the non-dimensional infinitesimal pa- 
rameter $t=a / L$, thus making the critical time $t_{c}$ equal to zero. The so-defined time plays the role of the first-order infinitesimal parameter in the asymptotic expressions for $\lambda, v, r$ and $d$.

The quantities introduced above depend on $s$ and $t$, and the following notations are used for the sake of brevity: $v(X, t)=v(s(X, a(t)), t)=v(s, t)$, $r(X, t)=r(s(X, a(t)), t)=r(s, t), d(X, t)=d(s(X, a(t)), t)=d(s, t)$.

It is shown in Nguyen (2000) that functions $\lambda(t), v(s, t), r(s, t)$ and $d(s, t)$ are governed by the following equation system:

- for $s \in] 0,1[$ :

$$
\begin{aligned}
& P(d) r "+t^{2} L^{2}=0 \\
& Q(d) r "+t^{2} L^{2}(\lambda r+v)=0(b) \\
& \dot{\lambda} r=\dot{v}-v^{\prime} \frac{s}{t}
\end{aligned}
$$

where the dot denotes the derivative with respect to time, $v^{\prime}$ and $r$ " stand for the first and second derivatives with respect to the space variable $s$, respectively. Functions $P(d)$ and $Q(d)$ are polynomials defined as:

$$
\begin{aligned}
& P(d)=-\int_{S}(d-Y) E_{i} d S=\frac{b}{8}\left[4\left(E_{T}-E\right) d^{2}+4\left(E_{T}+E\right) e d+\left(E_{T}-E\right) e^{2}\right] \\
& Q(d)=-\int_{S}(d-Y) Y E_{i} d S=\frac{b}{24}\left[-4\left(E_{T}-E\right) d^{3}+3\left(E_{T}-E\right) e^{2} d+\left(E_{T}+E\right) e^{3}\right]
\end{aligned}
$$

where $S$ is the cross-section of the beam and $E_{i}=E$ or $E_{T}$, depending on whether the considered point in the cross-section belongs to the elastic unloading zone or the plastic loading zone;

- for $s \in] 1,2[$ :

$$
\begin{aligned}
& E_{T} I r "+(1-t)^{2} L^{2}(\lambda r+v)=0(d) \\
& \dot{\lambda} r=\dot{v}-v^{\prime} \frac{2-s}{1-t}
\end{aligned}
$$

- furthermore, the boundary and connecting conditions write:

$$
\begin{array}{llll}
d(1, t)=\frac{e}{2} & (f) & r^{\prime}(0, t)=0 & (g) \\
v^{\prime}(0, t)=0 & (i) & v(2, t)=0 & (j) \\
r\left(1^{-}, t\right)=r\left(1^{+}, t\right) & (k) & v\left(1^{-}, t\right)=v\left(1^{+}, t\right)(l) \\
(1-t) r^{\prime}\left(1^{-}, t\right)=t r^{\prime}\left(1^{+}, t\right)(m) &
\end{array}
$$

- the initial conditions at $t_{c}=0$ are:
$\lambda(0)=\lambda_{c}$
$(n)$
$v(s, 0)=0$
$(o)$
$d(s, 0)=\frac{e}{2}$
$(p)$ 
The non-linear system $(a)-(p)$ is solved by the asymptotic expansion method, according to which the unknowns in hand are expanded into power series involving the non-dimensional small parameter $t$ :

$$
\begin{aligned}
& \lambda(t)=\lambda_{0}+\lambda_{1} t+\lambda_{2} \frac{t^{2}}{2}+\ldots \\
& v(s, t)=v_{1}(s) t+v_{2}(s) \frac{t^{2}}{2}+\ldots \\
& r(s, t)=r_{0}(s)+r_{1}(s) t+r_{2}(s) \frac{t^{2}}{2}+\ldots \\
& d(s, t)=d_{0}(s)+d_{1}(s) t+d_{2}(s) \frac{t^{2}}{2}+\ldots
\end{aligned}
$$

Let us explain in detail the calculations up to the second-order terms. The remaining terms can be addressed in the same way.

\section{$\underline{\text { Order } 0}$}

Equations of order 0 are $\left(P_{0} \doteq P(0), Q_{0} \doteq Q(0)\right)$ :

$$
\begin{array}{lll}
s \in] 0,1[: & P_{0} r{ }_{0}=0 \quad Q_{0} r{ }_{0}=0 & \lambda_{1} r_{0}=v_{1}-v_{1}^{\prime} s \\
s \in] 1,2[: & E_{T} I r{ }_{0}+L^{2} \lambda_{0} r_{0}=0 & \lambda_{1} r_{0}=v_{1}
\end{array}
$$

The boundary conditions are: $r_{0}^{\prime}(0)=0, r_{0}(2)=0$.

The connecting conditions are: $r_{0}\left(1^{-}\right)=r_{0}\left(1^{+}\right), r_{0}^{\prime}\left(1^{-}\right)=0$.

\section{$\underline{\text { Order } 1}$}

Equations of order 1 are $\left(P_{1} \doteq P_{,}(0), Q_{1} \doteq Q,{ }_{d}(0)\right)$ :

$$
\begin{aligned}
s \in] 0,1[: & P_{0} r{ }_{1}+P_{1} d_{1} r{ }_{0}=0 \\
& Q_{0} r{ }_{1}+Q_{1} d_{1} r{ }_{0}=0 \\
& \lambda_{2} r_{0}+\lambda_{1} r_{1}=v_{2}-v_{2}^{\prime} \frac{s}{2} \\
s \in] 1,2[: & E_{T} I r{ }_{1}+L^{2}\left(\lambda_{1} r_{0}+v_{1}+\lambda_{0} r_{1}-2 \lambda_{0} r_{0}\right)=0 \\
& \lambda_{2} r_{0}+\lambda_{1} r_{1}=v_{2}-v_{1}^{\prime}(2-s)
\end{aligned}
$$

The boundary conditions are: $d_{1}(1)=0, r_{1}^{\prime}(0)=0, r_{1}(2)=0, v_{1}^{\prime}(0)=0$, $v_{1}(2)=0$.

The connecting conditions are: $r_{1}\left(1^{-}\right)=r_{1}\left(1^{+}\right), v_{1}\left(1^{-}\right)=v_{1}\left(1^{+}\right), r_{1}^{\prime}\left(1^{-}\right)=$ $r_{0}^{\prime}\left(1^{-}\right)+r_{0}^{\prime}\left(1^{+}\right)$. 
$\underline{\text { Order } 2}$

Equations of order 2 are $\left(P_{2} \doteq P, d d(0), Q_{2} \doteq Q, d d(0)\right)$ :

$$
\begin{aligned}
s \in] 0,1[: & P_{0} r{ }_{2}+2 P_{1} d_{1} r{ }_{1}+\left(P_{1} d_{2}+P_{2} d_{1}^{2}\right) r{ }_{0}+2 L^{2}=0 \\
& Q_{0} r{ }_{2}+2 Q_{1} d_{1} r{ }_{1}+\left(Q_{1} d_{2}+Q_{2} d_{1}^{2}\right) r{ }_{0}+2 L^{2} \lambda_{0} r_{0}=0 \\
& \lambda_{3} r_{0}+2 \lambda_{2} r_{1}+\lambda_{1} r_{2}=v_{3}-v_{3}^{\prime} \frac{s}{3} \\
s \in] 1,2[: & E_{T} I r{ }_{2}+L^{2}\left(\lambda_{2} r_{0}+v_{2}+2 \lambda_{1} r_{1}-8 \lambda_{1} r_{0}+\lambda_{0} r_{2}-4 \lambda_{0} r_{1}+2 \lambda_{0} r_{0}\right)=0 \\
& \lambda_{3} r_{0}+2 \lambda_{2} r_{1}+\lambda_{1} r_{2}=v_{3}-\left(v_{2}^{\prime}+2 v_{1}^{\prime}\right)(2-s)
\end{aligned}
$$

The boundary conditions are: $d_{2}(1)=0, r_{2}^{\prime}(0)=0, r_{2}(2)=0, v_{2}^{\prime}(0)=0$, $v_{2}(2)=0$.

The connecting conditions are: $r_{2}\left(1^{-}\right)=r_{2}\left(1^{+}\right), v_{2}\left(1^{-}\right)=v_{2}\left(1^{+}\right), r_{2}^{\prime}\left(1^{-}\right)=$ $2\left(r_{1}^{\prime}\left(1^{-}\right)+r_{1}^{\prime}\left(1^{+}\right)\right)$.

Equations $(a)-(p)$ are solved sequentially, giving the $\lambda_{i}$ values together with the functions $v_{i}(s), r_{i}(s)$ and $d_{i}(s)$ involved in expansions (39). At order 0 , one gets $r_{0}(s)=C$ for $s \in[0,1]$, where $C$ is unknown so far. At order 1 , one gets $r_{0}(s)$ for $s \in[1,2]$, and the same expression for the critical stress $\lambda_{0}=\lambda_{T}$ as in (34). At order 2 , the value for constant $C$ is determined together with $v_{1}(s)=0$ for $s \in[0,2]$ and $\lambda_{1}=0$, and so on. Solving equations at order 3 provides $d_{1}(s)=0$ for $s \in[0,1], r_{1}(s)$ for $s \in[0,2], v_{2}(s)=0$ for $s \in[0,2]$ and $\lambda_{2}=0$. The calculations are carried out in a similar way up to the tenth order. The higher the considered order, the more intricate and lengthy the equations. Without going into details, we give the resulting load factor $\lambda$ and deflection $v$, as summarized in Tables 2 and 3 .

\begin{tabular}{|c|c|c|c|c|c|c|}
\hline$\lambda_{0}$ & $\lambda_{1}$ & $\lambda_{2}$ & $\lambda_{3}$ & $\lambda_{4}$ & $\lambda_{5}$ & $\lambda_{6}$ \\
\hline$\frac{\pi^{2} E_{T} I}{4 L^{2}}$ & 0 & 0 & 0 & 0 & $\frac{3 \pi^{6}\left(E-E_{T}\right) I}{16 L^{2}}$ & 0 \\
\hline
\end{tabular}

\begin{tabular}{|c|c|c|}
\hline$\lambda_{7}$ & $\lambda_{8}$ & $\lambda_{9}$ \\
\hline$-\frac{3 \pi^{8}\left(E-E_{T}\right)\left(36 E-25 E_{T}\right) I}{64 E_{T} L^{2}}$ & 0 & $\frac{9 \pi^{10}\left(E-E_{T}\right)\left(240 E^{2}-402 E E_{T}+173 E_{T}^{2}\right) I}{64 E_{T}^{2} L^{2}}$ \\
\hline
\end{tabular}

Table 2

Coefficients for load factor $\lambda$ in expansion (39)

Lastly, expressions for the plastic boundary $d_{i}(s=0)(i=0$ to 8$)$ in the expansion (39) can be obtained simultaneously with coefficients $\lambda_{i}$ and functions $v_{i}(s)$, as shown in Table 4. 


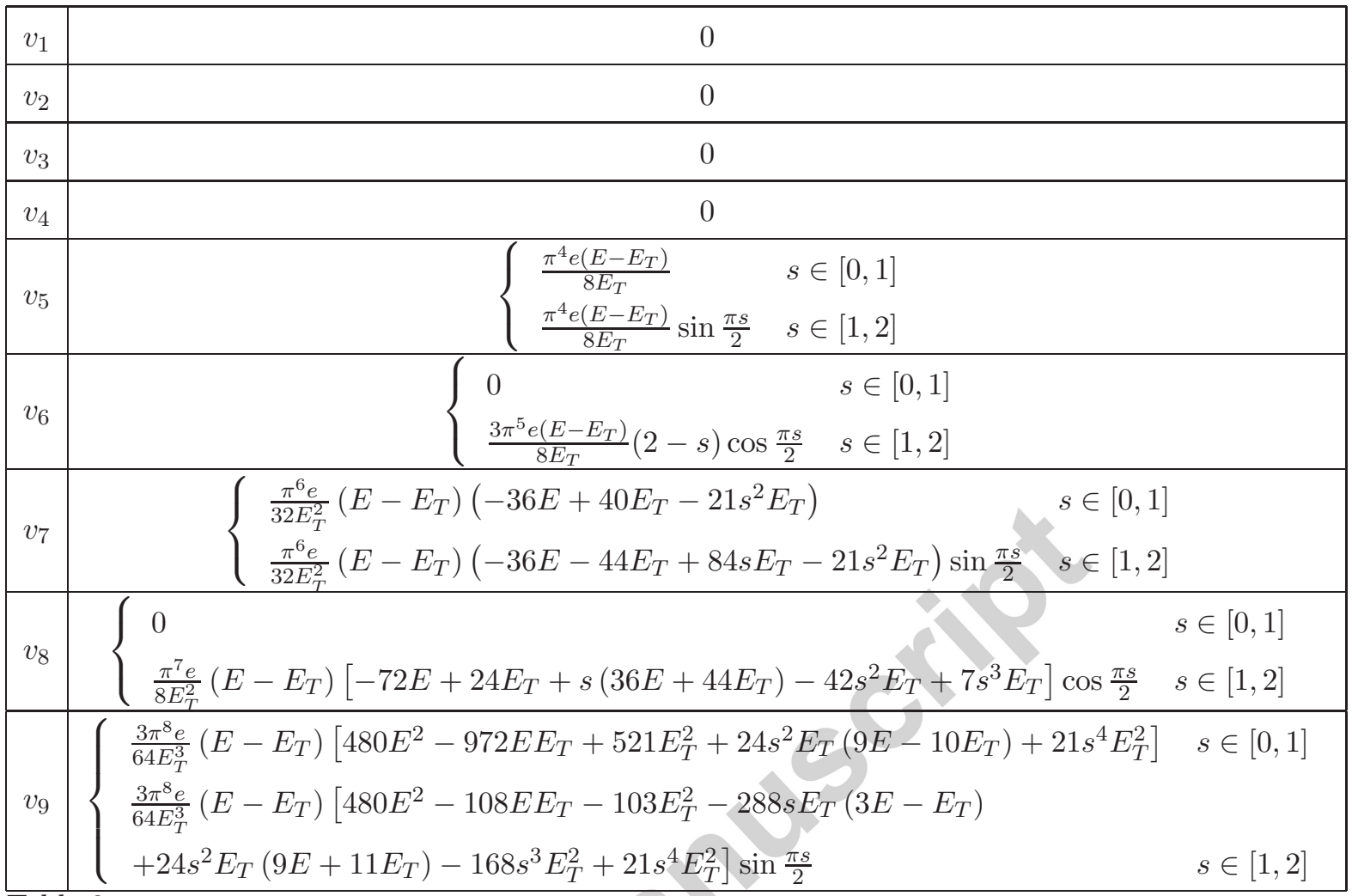

Table 3

Coefficients for deflection $v$ in expansion (39)

\begin{tabular}{|c|c|c|c|c|c|c|}
\hline$d_{0}(0)$ & $d_{1}(0)$ & $d_{2}(0)$ & $d_{3}(0)$ & $d_{4}(0)$ & $d_{5}(0)$ & $d_{6}(0)$ \\
\hline$\frac{e}{2}$ & 0 & $-\frac{\pi^{2} e}{8}$ & 0 & $\frac{\pi^{4} e}{32 E_{T}}\left(6 E-5 E_{T}\right)$ & 0 & $-\frac{\pi^{6} e}{256 E_{T}^{2}}\left(360 E^{2}-579 E E_{T}+221 E_{T}^{2}\right)$ \\
\hline
\end{tabular}

\begin{tabular}{|c|c|}
\hline$d_{7}(0)$ & $d_{8}(0)$ \\
\hline$-\frac{63 \pi^{6} e}{16 E_{T}}\left(E-E_{T}\right)$ & $\frac{\pi^{8} e}{512 E_{T}^{3}}\left(12600 E^{3}-29853 E^{2} E_{T}+22851 E E_{T}^{2}-5597 E_{T}^{3}\right)$ \\
\hline
\end{tabular}

Table 4

Coefficients for the plastic boundary $d(0, t)$ in expansion (39)

\subsection{Asymptotic expansion of the bifurcated branch}

From Tables 2 and 3, one can rewrite the truncated expansions for the load $\lambda$ and the maximum deflection-to-thickness ratio $\xi=\frac{2 v(0, t)}{e}$ as follows:

$$
\begin{aligned}
& \lambda=\lambda_{0}+\lambda_{5} \frac{t^{5}}{5 !}+\lambda_{7} \frac{t^{7}}{7 !}+\lambda_{9} \frac{t^{9}}{9 !} \\
& \xi=\xi_{5} \frac{t^{5}}{5 !}+\xi_{7} \frac{t^{7}}{7 !}+\xi_{9} \frac{t^{9}}{9 !}
\end{aligned}
$$

where $\xi_{i}=\frac{2 v_{i}(0)}{e}$ for $i=5,7,9$. 
By eliminating parameter $t$ from relations (40) in a step-by-step manner, one gets the following asymptotic expansion with fractional exponents for the load $\lambda$ with respect to parameter $\xi$ :

$$
\lambda=\lambda_{0}\left(1+c_{1} \xi+c_{2} \xi^{\frac{7}{5}}+c_{3} \xi^{\frac{9}{5}}+o\left(\xi^{\frac{9}{5}}\right)\right)
$$

where:

$$
\begin{gathered}
c_{1}=3 \quad c_{2}=-\frac{15}{7}\left(\frac{15 \pi \frac{E_{T}}{E}}{4 \sqrt{2}\left(1-\frac{E_{T}}{E}\right)}\right)^{\frac{2}{5}} \\
c_{3}=\frac{65 \frac{E_{T}}{E}-48}{42 \frac{E_{T}}{E}}\left(\frac{15 \pi \frac{E_{T}}{E}}{4 \sqrt{2}\left(1-\frac{E_{T}}{E}\right)}\right)^{\frac{4}{5}}
\end{gathered}
$$

Expressions for $c_{1}$ and $c_{2}$ agree well with those proposed by Cheng, $\mathrm{Lu}$ and Fang (1995). On the other hand, our expression for $c_{3}$ slightly differs from that of Cheng, Lu and Fang, which is $\frac{17}{42}\left(\frac{15 \pi \frac{E_{T}}{E}}{4 \sqrt{2}\left(1-\frac{E_{T}}{E}\right)}\right)^{\frac{4}{5}}$. They would be the same if one made $E_{T}=E$ in the first ratio in (42).

It should be noted that the fractional expansion (41) is only valid if the maximum deflection-to-thickness ratio $\xi=\frac{2 v(0, t)}{e}$ is small, in the same way as the time parameter $t=a / L$ related to it by the second relation in (40). Let us consider, as an example, the validity domain in $\xi$ for a beam with $E / E_{T}=2.4$ (the same material as in Subsection 3.3).

Figure 4 displays $\xi$ versus $t$ and $\lambda / \lambda_{T}$ versus $\xi$, making use of relations (40)(42). As can be seen in Figure 4(a), at the beginning of the bifurcation process, the elastic unloading region with length $a$ spreads out very quickly up to about a third of the beam length, while $\xi$ remains very small, of the order of 0.001. Moreover, Figure 4(b) shows that the fractional expansion up to the 9/5-order (41) can be identified to the parametric expansion up to the ninth order (40) with parameter $t$ if $\xi$ is small enough. Thus, in the sequel, we will limit ourselves to $\xi \leqslant 0.01$ (corresponding to $t \leqslant 0.51$ ), so that the difference between the fractional and parameter expansions do not exceed $0.7 \%$.

\subsection{Finite element validation}

Several finite element computations are presented hereafter in order to check the previous analytical results obtained for the plastic post-buckling of a beam. The numerical computations are conducted on a simply-supported beam, which actually gives the same results as a cantilever beam because of symmetry. The geometry and material data are given in Table 5 with the notations 


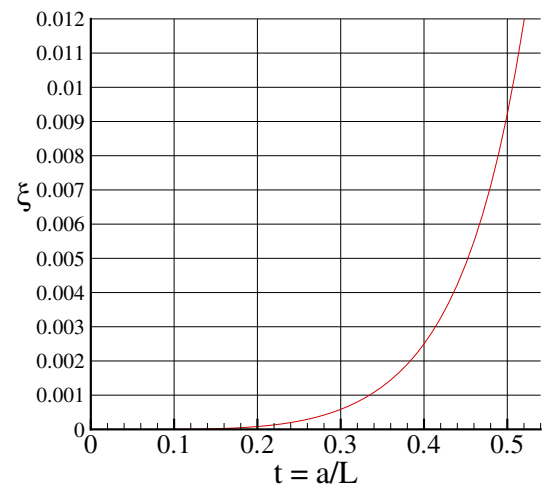

(a)

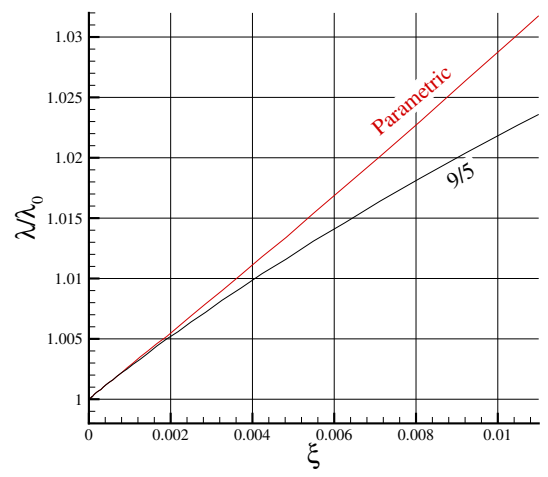

(b)

Fig. 4. Validity of expansion (41) in terms of the maximum deflection-to-thickness ratio $\xi=\frac{2 v(0, t)}{e}$

defined in Figure 3. One tip is fixed in the $\mathbf{x}$-direction and the other is loaded by a uniform compressive force $\lambda$. Only the middle node at each tip is fixed in the z-direction, which enables a uniform stress state in the beam during the pre-critical deformations. Since the thickness is very thin, the numerical solution of the bending problem can be performed using the shell finite element described in Le Grognec and Le van (2008). One single finite element in the $\mathbf{z}$-direction is used, whereas 50 elements along the length are necessary to correctly represent finite rotations. Use is made of two Gaussian points in the $\mathbf{x}$ and $\mathbf{z}$-directions and four Gaussian points through the thickness $\mathbf{y}$-direction. Use is made of the same computer program as in Subsection 3.3. Here, one also needs additional branch-switching and arc-length methods (Riks, 1979, 1991; Lam and Morley, 1992; Seydel, 1994) in order to bifurcate from a specific point of the continuous bifurcation range and pass through load or displacement limit points on the bifurcated paths.

Table 5

\begin{tabular}{|c|c|c|c|c|c|}
\hline$L$ & $e$ & $b$ & $E$ & $E_{T}$ & $\sigma_{0}$ \\
\hline $0.5 \mathrm{~m}$ & $1 \mathrm{~mm}$ & $5 \mathrm{~cm}$ & $2.110^{11} \mathrm{~Pa}$ & $8.7510^{10} \mathrm{~Pa}$ & $5.10^{4} \mathrm{~Pa}$ \\
\hline
\end{tabular}

Data for the finite element computation of the bifurcated branch

The material is the same as in Subsections 3.3 and 4.2, except for the yield stress: as the beam is very thin in the $\mathbf{y}$-direction, $\sigma_{0}$ has been given a very small value for the plastification to occur before the beam buckles at the tangent modulus critical load $\lambda_{T}$. The ratio $E / E_{T}=2.4$ is small enough to obtain separate continua of bifurcation points and simplify the analysis. In the case of a rectangular cross-section, the so-called reduced modulus can be 
expressed as follows:

$$
E_{R}=\frac{4 E E_{T}}{\left(\sqrt{E}+\sqrt{E_{T}}\right)^{2}}=1.2910^{11} \mathrm{~Pa}
$$

In each continuous range of bifurcation points, the critical loads corresponding to the three moduli - the tangent modulus, the reduced modulus and the elastic modulus - are in ascending order:

$$
\lambda_{T n}=\frac{n^{2} \pi^{2} E_{T} I}{4 L^{2}} \quad \lambda_{R n}=\frac{n^{2} \pi^{2} E_{R} I}{4 L^{2}} \quad \lambda_{E n}=\frac{n^{2} \pi^{2} E I}{4 L^{2}}
$$

whence the numerical values for the first mode:

$$
\lambda_{T 1}=3.6 N \quad \lambda_{R 1}=5.3 N \quad \lambda_{E 1}=8.64 N
$$

\subsubsection{Elastoplastic bifurcation diagrams}

In plasticity, each point of the continuous bifurcation range is a potential bifurcation point, and the corresponding non-trivial rate solution involves an elastic unloading zone whose shape depends on the compressive force value. At the tangent modulus critical load, the concept of "comparison elastic solid" allows one to use the same approach as in elasticity: the critical load $\lambda_{T}$ is detected when the stiffness matrix becomes singular and the bifurcated branch (which starts with a single unloading point) can be predicted by the corresponding eigenmode. If one wishes to bifurcate at another point in the continuous range of bifurcation points, the prediction is based on the eigenmode corresponding to the smallest eigenvalue of the tangent stiffness matrix.

The obtained numerical results show that this procedure enables one to correctly bifurcate in the whole continuous range of bifurcation points. In agreement with the well-known theoretical results, the obtained curves are bounded by those corresponding to the tangent modulus critical load $\lambda_{T}$ and the elastic critical load $\lambda_{E}$. Figure 5 plots the maximum displacement at the middle of the beam versus the compressive force. It can be seen that the bifurcation takes place with an increasing load when $\lambda_{c} \in\left[\lambda_{T}, \lambda_{R}\right.$ [, with a decreasing load when $\left.\left.\lambda_{c} \in\right] \lambda_{R}, \lambda_{E}\right]$, and with a constant load at $\lambda_{R}$, as predicted by the theory.

In the initial post-critical zone (small deflections), say $V<0.0005 m$ in Figure 5 (a), all the bifurcated branches converge towards the asymptotic curve $\lambda=$ $\lambda_{R}$, as predicted by the analytical solution of El Koulani (1996). However, Figure 5(b) shows that, in the advanced post-critical zone where the deflections are larger and the geometric non-linearities prevail, the bifurcated branches tend towards another asymptote. 


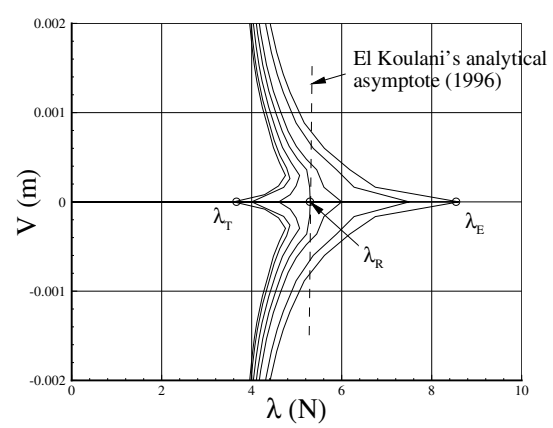

(a) Initial post-buckling

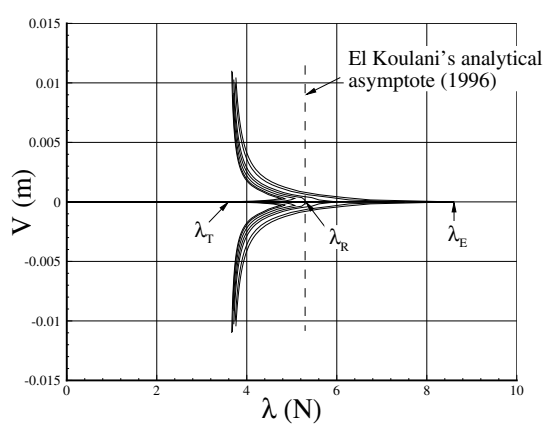

(b) Advanced post-buckling

Fig. 5. Elastoplastic bifurcation diagrams

Let us now focus on the bifurcated branch arising from the first tangent modulus critical load $\lambda_{T 1}$. Figure 6 displays four different post-critical curves $\lambda=\lambda(\xi)$ :

- the curve obtained from the finite element results;

- the analytical fractional curve (41) including two non-linear terms with exponents $7 / 5$ and $9 / 5$;

- for comparison purposes, the fractional curves with only the linear term and with the 7/5-order term, as obtained by Hutchinson (1974).

All the fractional curves are drawn for $\xi \leqslant 0.01$, in accordance with the validity domain in $\xi$, as discussed in Subsection 4.2. Figure 6 shows that the finite element results are in very good agreement with the fractional expansion (41). Over the considered range of $\xi$, the difference is less than $0.1 \%$ in absolute value.

\subsubsection{Plastic boundary and unloading zone}

Let us focus now on the evolution of the shape of the unloading zone in the beam along the bifurcated branch arising from the tangent modulus critical load.

Again, consider a simply-supported beam of length $l=2 L=1 \mathrm{~m}$. Here, in order to make the unloading zone visible, let us now take the height $e=10 \mathrm{~cm}$ along the $\mathbf{y}$-axis, the width $b=1 \mathrm{~cm}$ along the $\mathbf{z}$-axis, and enforce the bending to take place in the $\mathbf{x y}$-plane by prescribing all the out-of-plane degrees of freedom to zero, see Figure 7.

The material is the same as before, except for $\sigma_{0}$ which is again $5.10^{7} \mathrm{~Pa}$. The mesh for the new geometry involves 30 elements along the $\mathbf{x}$-direction and 4 elements along the $\mathbf{y}$-direction. In each element, use is made of seven 


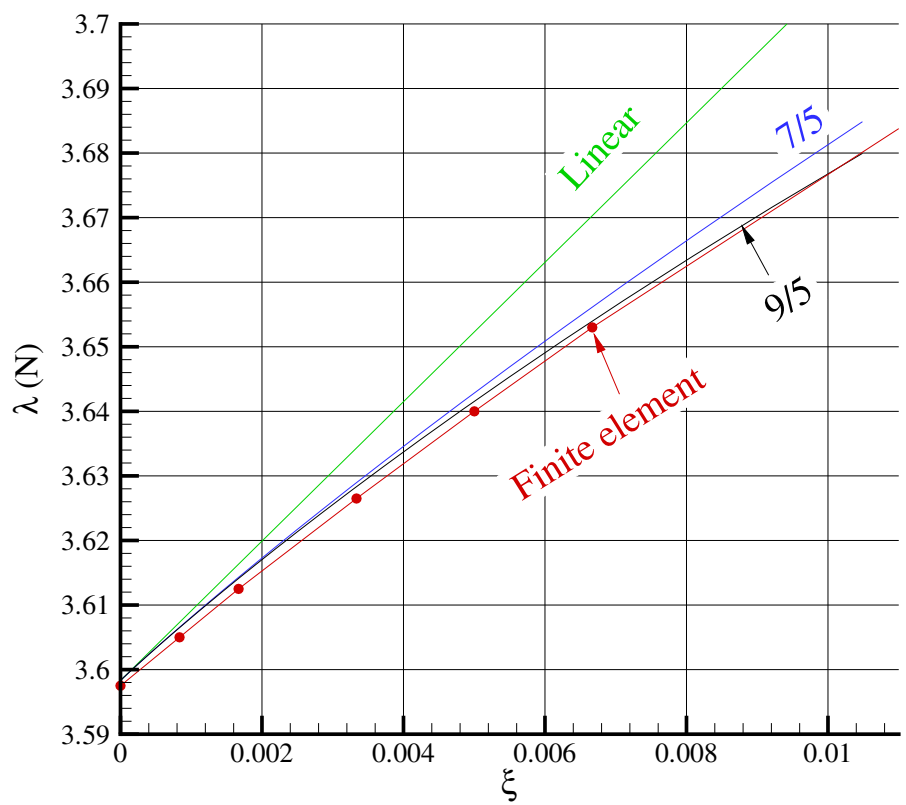

Fig. 6. Tangent modulus bifurcation: comparison between different fractional expansions and the finite element results. Linear: curve with only the linear term in (41); $7 / 5$ : curve with terms up to exponent $7 / 5 ; 9 / 5$ : curve with terms up to exponent $9 / 5$

\section{$\lambda$}

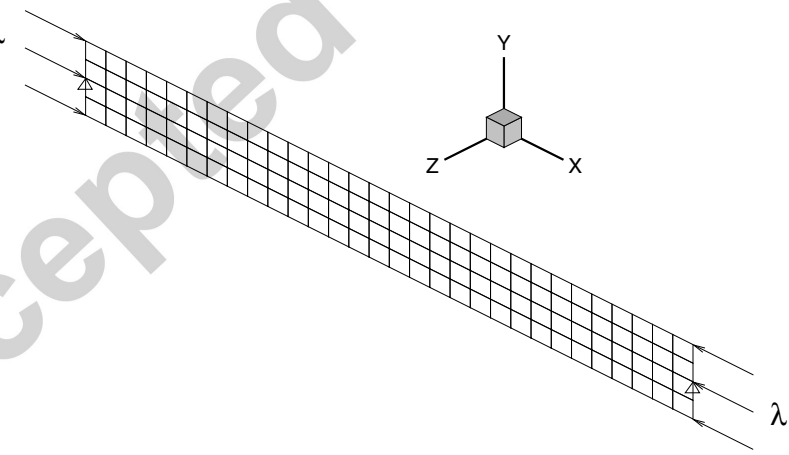

Fig. 7. Geometry, mesh and boundary conditions for the analysis of the unloading zone

Gaussian points in both $\mathbf{x}$ - and $\mathbf{y}$-directions. Only two Gaussian points along the $\mathbf{z}$-direction are used for the numerical integration through the thickness, as the elastoplastic state is uniform along that direction. In theory, the plastic points are those with positive plastic strain rates. Here, in the numerical computations, they are detected in a discrete way: since the unloading zone keeps 
growing (at least in the vicinity of the bifurcation point), the plastic points at increment $n$ are numerically detected by comparing the equivalent plastic strains between steps $n$ and $n+1$.

At the tangent modulus critical load, the unloading zone spreads out from a single point, as one advances on the bifurcated branch. Applying the procedure above, the unloading zones obtained by numerical computations (symmetric with respect to the median plan of the beam) are in very good agreement with the theoretical ones, as shown in Figure 8 for a same load level.
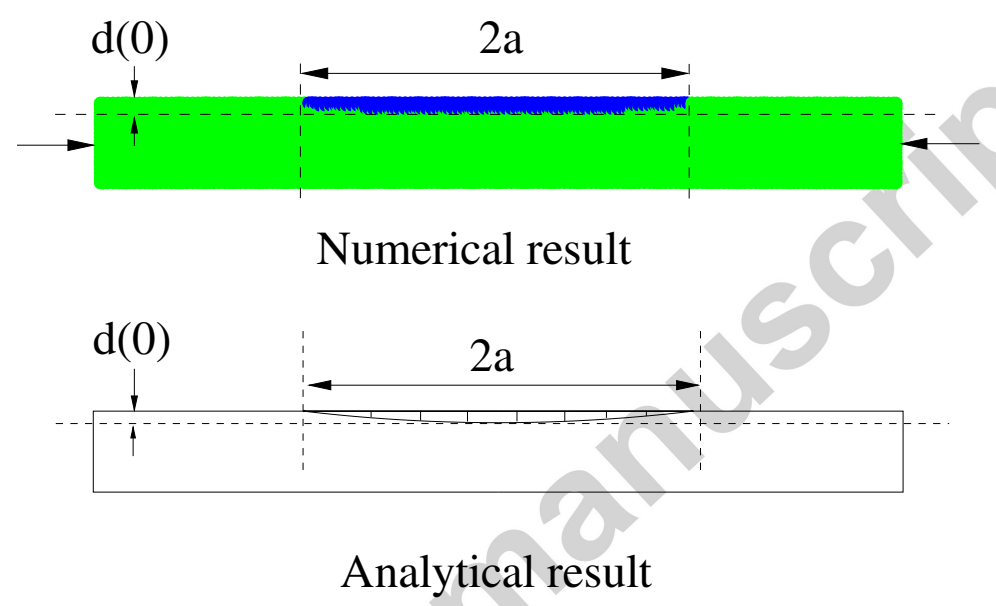

Fig. 8. Unloading zone for the bifurcated branch arising from the tangent modulus critical load (first mode)

\section{Conclusions}

Two new issues have been investigated in the field of the elastoplastic buckling and post-buckling of beams under axial compression.

(i) The first issue is the plastic bifurcation of a Timoshenko beam, where the transverse shear effects are taken into account. The analysis is based on the 3D plastic bifurcation theory and proves to be an efficient way to deal with structures subjected to a uniform uniaxial pre-critical stress state, as shown in an earlier study on compressed plates and cylinders (Le Grognec and Le van, 2009). Here, the same 3D approach has been applied to a Timoshenko beam and enabled one to obtain the tangent modulus critical load, the buckling mode and the initial slope of the bifurcated branch. The transverse shear effects may be significant depending on the cross-section geometry, as clearly 
shown in the finite element computation with a hollow rectangular section, where the Timoshenko theory gives better results than the Euler-Bernoulli one.

(ii) The second issue of the paper is a further analysis of the post-bifurcation of a Euler-Bernoulli beam, stemming from the tangent modulus bifurcation. Use has been made of a specific method in order to provide the fractional expansion of the post-critical branch, exhibiting one new non-linear term. Also, the shape of the elastic unloading region in the beam during the bifurcation process has been obtained in the form of an asymptotic expansion. The finite element computations are in very good agreement with the presented analytical results.

Two conclusions may be drawn from the fractional expansion study of the post-bifurcated branch:

- Compared to the 7/5-order term, the 9/5-order one brings about a slight change and a better description of the post-critical behavior.

- For all practical purposes, there would be no need to go further than the $9 / 5$-order term in the fractional expansion.

\section{References}

Akel, S., On the buckling of elastoplastic structures (Sur le flambage des structures élastoplastiques), PhD Thesis (Ecole Nationale des Ponts et Chaussées, France, 1987).

Cheng, Y.S., Lu, W.D. and Fang, H., Plastic post-buckling of a simplysupported column with a solid rectangular cross-section, Applied Mathematics and Mechanics 16 Issue 8 (1995) 713-722.

Cheng, Y.S., Fang, H. and Lu, W.D., Imperfection sensitivity analysis of a rectangular column compressed into the plastic range, Applied Mathematics and Mechanics 19 Issue 1 (1998) 9-14.

Cimetière, A., On the modelling and buckling of elastoplastic plates (Sur la modélisation et le flambage des plaques élastoplastiques), PhD Thesis (University of Poitiers, France, 1987).

Cimetière, A., El Koulani, A. and Léger, A., Buckling and post-buckling of an elastoplastic simple model (Flambage naissant et post-flambage d'un modèle simple en élastoplasticité), Comptes Rendus de l'Académie des Sciences (Paris t. 319, 1994).

Durban, D., Plastic buckling of plates and shells, Stability analysis of plates and shells (NASA CP-1998-206280, 1998) 293-311.

El Koulani, A., Continuation in moving boundary problems of the plastic bifurcation type (Continuation dans les problèmes à frontières libres de type bifurcations plastiques), PhD Thesis (University of Metz, France, 1996).

El Koulani, A. and Léger, A., Bifurcated solutions of the initial velocity prob- 
lem for an elastoplastic beam (Solutions bifurquées du problème en vitesses initiales pour une poutre élastoplastique), Comptes Rendus de l'Académie des Sciences (Paris t. 322, 1996).

El Koulani, A. and Léger, A., Post-critical behavior of elastoplastic beams: existence and regularity of the bifurcated branches (Comportement postcritique des poutres élastoplastiques : existence et régularité des branches bifurquées), Comptes Rendus de l'Académie des Sciences (Paris t. 324, 1997).

Hill, R., A general theory of uniqueness and stability in elastic-plastic solids, Journal of the Mechanics and Physics of Solids 6 (1958) 236-249.

Hutchinson, J.W., On the post-buckling behavior of imperfection-sensitive structures in the plastic range, Journal of Applied Mechanics 39 (1972) $155-162$.

Hutchinson, J.W., Plastic buckling, Advances in Applied Mechanics 14 (1974) $67-144$.

Lam, W.F. and Morley, C.T., Arc-length method for passing limit points in structural calculation, Journal of Structural Engineering 118 Issue 1 (1992) 169-185.

Le Grognec, P. and Le van, A., A unified treatment for the elastoplastic bifurcation of structural elements, 21 ${ }^{\text {st }}$ International Congress of Theoretical and Applied Mechanics Warsaw, Poland (August 15-21, 2004).

Le Grognec, P. and Le van, A., Elastoplastic bifurcation and collapse of axially loaded cylindrical shells, International Journal of Solids and Structures 45 Issue 1 (2008) 64-86.

Le Grognec, P. and Le van, A., Some new analytical results for plastic buckling and initial post-buckling of plates and cylinders under uniform compression, Thin-Walled Structures 47 Issues 8-9 (2009) 879-889.

Nguyen, Q.S., Bifurcation and stability of irreversible systems obeying to the maximum dissipation principle (Bifurcation et stabilité des systèmes irréversibles obéissant au principe de dissipation maximale), Journal de Mécanique Théorique et Appliquée 3 Issue 1 (1984) 41-61.

Nguyen, Q.S. and Stolz, C., On the asymptotic development method in plastic buckling (Sur la méthode de développement asymptotique en flambage plastique), Comptes Rendus de l'Académie des Sciences (Paris t. 300, 1985).

Nguyen, Q.S., Stability and Non-Linear Solid Mechanics (Wiley, 2000).

Riks, E., An incremental approach to the solution of snapping and buckling problems, International Journal of Solids and Structures 15 Issue 7 (1979) $529-551$.

Riks, E., On formulations on path-following techniques for structural stability analysis, New advances in computational structural mechanics (Elsevier, 1991) 65-80.

Sewell, M.J., A survey of plastic buckling, Stability (University of Waterloo Press, 1972) 85-197.

Seydel, R., Practical bifurcation and stability analysis. From equilibrium to chaos (Springer-Verlag, 1994).

Shanley, F.R., Inelastic column theory, International Journal of Aeronautical 
Sciences 14 (1947) 261-267.

Tvergaard, V. and Needleman, A., On the buckling of elastic-plastic columns with asymmetric cross-sections, International Journal of Mechanical Sciences 17 (1975) 419-424. 